mals which could be read to advantage by both major and minor scholars.

R. J. HARRISON

\section{Natural Nerve Poisons}

Neuropoisons: Their Pathophysiological Actions. Vol. 1: Poisons of Animal Origin. Edited by Lance L. Simpson. Pp. xiv +361 . (Plenum: New York and London, 1971.) \$22.50.

RESEARCH workers interested in the actions of neurotoxins, or contemplating the use of these substances as tools for the analysis of neuro-physiological function, should find this book useful as an introduction to the subject. By presenting evidence of modes of action it may also help clinicians to devise rational therapy for accidental victims of the poisons. The book is an edited collection of 15 chapters, by 17 contributors, covering the marine poisons tetrodotoxin and saxitoxin, the low molecular weight protein neurotoxins from elapid and crotalid snake venoms and the high molecular weight tetanus and botulinum toxins. Although the emphasis is on the neurotoxins, several chapters also deal with other actions such as the cardiovascular, haemolytic and coagulant effects of the snake venoms and the botulinum haemagglutinin.

As each chapter is written by a different author, it is unavoidable that some unevenness is noticeable, but it has probably been reduced by editing. Editorial control also appears to have avoided a lot of overlapping between chapters, except for some repetitive duplication in chapters 7,8 and 9 . Many of the chapters are well balanced detailed reviews of published (and occasional unpublished) work. A few authors have contributed more personal summaries centred around their own work. I was particularly pleased to find that Yasumi Ogura had contributed a chapter on tetrodotoxin and puffer-fish poisoning. $\mathrm{He}$ has made many contributions but most of them are written in Japanese, or are otherwise difficult of access, and his chapter provides a very useful guide to this and to other Japanese work that will be of help to English readers.

The most serious criticism to be made of the book is that many of the references are inaccurate. In one or two chapters these inaccuracies are so many that the only possible explanation is that the manuscript was carelessly prepared. Some of the citations in the text are not listed among the references, or appear under a different date. More serious are the occasional references to an irrelevant publication. This sort of careless error tends to reduce the value of the work for those who wish to use it as an introduction to the field. Most of the chapters are well illustrated, generally with figures taken from other publications. The line drawings have reproduced well. The half-tone oscillograms are unorthodox with their grey backgrounds but are perfectly satisfactory except for a slight loss of detail when compared with the originals in the Proceedings of the Royal Society. Some of the half-tone photographs, however, are unsatisfactory.

In spite of these criticisms the book is a valuable review of a field whose importance to the neurobiologist is increasing rapidly. It would have been pedantic not to have included saxitoxin in this volume because, although it originates in phytoplankton, it is pharmacologically almost identical with tetrodotoxin. One looks forward to the publication of the second volume, dealing with neuropoisons of plant origin, and hopes that the price will not be greater than the already rather high cost of the first volume.

\section{MaRTIN H. Evans}

\section{Physics Without Tears}

Physics from the Ground Up. By Herman Carr and Richard T. Weidner. Pp. xiv +706 . (McGraw-Hill: New York and Maidenhead, April 1971.) $£ 6.95$.

IN the past few years, universities and colleges in the United States have produced a remarkable number of introductory physics texts, designed for intelligent students who know little physics from school and who are unlikely to become professional scientists. These books have a number of characteristics in common. They are lavishly produced and rarely run to less than 500 pages, they treat physics from the beginning but at an adult level, they concentrate on the fundamentals at the expense of applications and they rarely use calculus.

This volume fits firmly into this category and must be judged against others of the same genre. On the whole, it is more down to earth, but for that very reason less likely to produce unexpected insights or to illuminate the subject from an unusual angle; it uses two colours to excellent pedagogic and aesthetic effect, its illustrations are outstanding and it has a most useful set of worked problems. In summary, it is a very good and useful example of its kind, and better than many.

There are a number of topics that one can use as touchstones for good textbooks, and on these the present book comes out well. It has a thoughtful treatment of inertial mass, it deals with the hydrogen atom without using either Bohr orbits or standing waves in a circle and it explains Bragg "reflection" clearly and accurately. These are points worth noting.
On the selection of subject matter, I am struck by the fact that it has the datedness of the fashion that has just been superseded. The text is said to fall into five main areas-classical conservation laws, classical interactions, light and waves, the very fast, the very small. The theme is clearly "the physics of elementary particles and all that is needed to lead up to it". But one has grown a little disillusioned with the frontier of science approach to physics textbooks, with the last chapter giving a necessarily confused account of our confused state of knowledge of elementary particles. There is nothing, for instance, on the solid state, liquids or aerodynamics ; in other words, there is little or nothing that makes one feel that physics is useful and linked with everyday life.

And yet it is a good book to have around. It is obviously too expensive for English students to buy and it also deals with a pedagogical problem that we do not normally attempt to tackle -that of starting physics at college level. It deserves a place on the shelf of both the sixth form and undergraduate library and it should join the growing band of American textbooks which sooner or later ought to persuade us that to teach physics to the nonphysics major is possible and can even be exciting.

I. R. B. Elton

\section{Stellar Atmospheres Clarified}

Stellar Atmospheres. By Dimitri Mihalas. (Series of Books in Astronomy and Astrophysics.) Pp. xiv +463 . (Freeman: San Francisco and Reading, February 1971.) $£ 7.50$.

THE central problem in the theory of stellar atmospheres is to relate "theoretician's" quantities such as total luminosity, effective temperature, surface gravity and chemical composition to "observer's" quantities such as broadband and narrow-band magnitudes and the intensities and profiles of spectral features. It is because the latter depend on details of radiative transfer in the outer mass shell of a few $\mathrm{g} \mathrm{cm}^{-2}$ from which radiation escapes to space and to the observer that the small "tail" of a star formed by its atmospheric layers assumes an importance out of all proportion to its mass and is being studied with computers and other means as actively as the theory of stellar evolution itself. This problem of radiative transfer is not by any means the only interesting problem connected with stellar atmospheres, but other questions (such as the origin of chromospheres, coronas and the solar cycle) are outside the scope of the book under reviewa reasonable limitation in view of the 